\title{
Sugar substitutes and health
}

\author{
Dr.Pramod Yadav ${ }^{1}$, Dr.Bakshish Kaur ${ }^{2}$, Dr.Ruchi Srivastava ${ }^{3}$, Dr.Sumedha \\ Srivastava ${ }^{4}$ \\ ${ }^{I}$ (Public Health Dentistry, K.D.Dental College \&Hospital/Agra University, India) \\ 2 (Prosthodontics, Peoples College of Dental Sciences/Peoples University, India) \\ ${ }_{3}^{3}$ (Periodontics, Saraswati Dental College, Lucknow/Dr.RML Avadh University, India) \\ ${ }^{4}$ (Periodontics Peoples College of Dental Sciences/Peoples University, India)
}

\begin{abstract}
The sugar substitutes also may also be called as alternative, artificial, high-intensity, or nonnutritive sweeteners, can replace the sweetness of sugar while providing few or no calories. In addition to the calorie savings, these sugar substitutes have the advantage of not promoting tooth decay, and they are useful in dietary planning for people who are coping with obesity or diabetes. Sugar substitutes includes both food ingredients with very strong sweetening power that provide zero or very few calories, which are used in very small amounts to sweeten foods, and bulk sweetening agents such as polyols, which can replace both the bulk of sugar and some of its sweetness. The availability of a variety of safe sugar substitutes is of benefit to consumers because it enables food manufacturers to formulate a variety of good-tasting sweet foods and beverages that are safe for the teeth and general health.
\end{abstract}

Keywords: artificial-sweeteners, aspartame, sugar-substitutes, xylitol, sucrose.

\section{Introduction}

The process of dental caries is one of the oldest and ubiquitous diseases of the prehistoric and modern times. Hundreds of dental research investigators like Miller, Gottleib, Keye for more than a century have studied various aspects of dental caries etiopathogenesis. Despite the extensive investigation many aspects of etiology are still obscure though many theories have been postulated from time to time. ${ }^{1}$

Dental caries is still a major problem across the world affecting $60-90 \%$ of school children and adults (WHO 2003). The Indian scenario is no better with a caries prevalence rate of $83.4 \%$ amongst the $15 \mathrm{yr}$ old group while $83-86 \%$ in $35-44 \mathrm{yr}$ old population. As dental professionals it is our prime concern to check this increasing incidence of dental caries and adopt various means to reduce it.

According to the currently accepted concept caries is truly a multifactorial disease. Interaction between three primary factors namely host tissue-the tooth, microflora with cariogenic potential and suitable local substrate i.e. diet; is essential for initiation of caries disease process (Keye1960). However, Newbrun (1982) added a fourth factor "time" to the three above factors and the concept came to be known as "caries tetralogy". Saliva is also considered an important factor as teeth are in continuos contact with saliva. ${ }^{1}$

From time to time various studies have been done to prove the indispensability of each of the contributory factors of caries process. Kite et al (1950) established the fact that rats fed by stomach tube do not develop caries despite the prevalence of cariogenic microflora. Historical studies of Hopewood house also concluded that caries in children can be greatly reduced by a Spartan diet which includes rigid restriction of refined carbohydrates or sugars. These studies implicating sugars as primary factor for caries formation are truly overwhelming and have established dietary concepts of decay.

In a series of collaborative studies carried out in Turku, in Finland by Schenin et al(1975), cariogenic potential of sucrose, fructose and xylitol was compared. Fructose was found to be as cariogenic as sucrose while xylitol produced anticariogenic effects. ${ }^{3}$

No doubt, it is difficult to avoid sugar in diet but reducing the amount and exposure to sugar in diet of humans especially children is an important consideration in preventing caries thus non-cariogenic sweeteners offer good alternative to sugar if used in moderation. These sugar substitutes do not promote caries.

As dental professionals we have the responsibility to reduce the incidence of dental caries by emphasizing the role of sugars in caries formation. It is important that the dentist must be familiarised with alternatives to sugar and type of food products that are available with substitute sweetening agents.

\section{Classification Of Sugar Substitutes}

Based on sugar substitute being caloric or non-caloric:

a) Caloric / Nutritive sweetener

i) Poly alcohols / sugar alcohols b) Non caloric / Non nutritive sweetener

i) Cyclamate 
- Xylitol

- Sorbitol

ii) Hydrogenated starch hydrolysate

- Lycasin

- Palatinit

iii) Coupling sugar

- Sorbose

- Palatinose

Based on their origin:

A)Natural (derived from plant origin)

1. Monellin

2. Licorice

3. Dihydrochalcone

4. Miraculin ii) Saccharin

iii) Aspartame

iv) Sucralose

v) Neotame

B)Artificial

1. Aspartame

2. Saccharin

3. Cyclamate

4. Sucralose

Based On Sugar Substitutes being caloric or non-caloric:

Sweetening agents proposed or used to replace acidogenic sugar as a means to reduce dental caries incidence can be divided into two broad categories:

\section{CALORIC /NUTRITIVE SWEETNERS}

- The caloric sweeteners provide 4 calories per gram.

- Caloric sweeteners provide sweet flavor and bulk when added to food.

- They also maintain freshness and contribute to product quality.

- Caloric sweeteners act as a preservative in jams and jellies, and a flavor enhancer in processed meats.

- They provide fermentation for breads and pickles, bulk to ice cream, and body to carbonated beverages.

Some caloric sweeteners are made by processing sugar compounds and some occur naturally. They are further divided into three types :

i. Polyalcohols /sugar alcohols: These are not sugars but derivatives of sugars in which the reactive aldehyde or keto group is reduced to hydroxyl group.

ii. Hydrogenated Starch Hydrolysate These are derived from starch by partial hydrolysis and subsequent hydrogenation.

iii. Coupling Sugars: By enzymatic treatment of starch, a mixture of monosaccharides, glucosylsucrose, maltosylsucrose and oligosac-charides can be produced, called coupling sugars.

\section{Examples}

SUGAR ALCOHOLS

Xylitol

Sorbitol

Maltitol
STARCH-HYDROLYSATES

Lycasin

Palatinit
COUPLING SUGARS

Sorbose

Palatinose

\section{NON CALORIC /NON NUTRITIVE SWEETNERS}

The noncaloric sweetners provide zero calories but they do provide the sweet taste. Noncaloric sweeteners are used in place of caloric sweeteners in some cases. All noncaloric sweeteners are chemically processed. They are:

- Cyclamate

- Saccharin

- Aspartame

- Sucralose

- Neotame

\section{Various Available Sugar Substitutes}

I.Caloric /Nutritive Sweetners

\section{I.1Sugar alcohols (Polyalcohol):}

Polyalcohol such as sorbitol and xylitol are important sugar substitutes since they are not efficient substrates for plaque bacteria and therefore produce only a minimal drop in plaque $\mathrm{pH}$. 


\section{1A: Sorbitol}

Metabolism of sorbitol in streptococcus mutans.

It was introduced in the diet of diabetics as early as 1929. In the 1970's it also made a mark in the field of dentistry when various experiments were conducted to check the cariogenicity of sorbitol Though S. mutans have enzymes to degrade sorbitol, the fermentation process is slow and hence very little drop in $\mathrm{pH}$ of dental plaque was recorded after the ingestion of sorbitol as compared to the rapid drop following the use of sucrose. ${ }^{2}$

\section{Side effects of sorbitol:}

Ingesting large amounts of sorbitol can lead to some abdominal pain, gas, and mild to severe diarrhoea. Sorbitol can also aggravate irritable bowel syndrome and fructose mal absorption ${ }^{32}$.

\section{I.1B Xylitol:}

Metabolism of Xylitol in Xylitol-Fermenting Strains of Lactobacilli:

In September, 1890, the German chemistry professor Emil Herman Fischer and his assistant, Rudolf Stahel, separated from beech chips a new compound which was named Xylit, the German word for Xylitol. Xylitol was used initially by diabetics as its metabolism was considered to be insulin-independent. ${ }^{2}$

The anti- cariogenic potential of Xylitol was first of all established in the landmark study of Turku in Finland in 1975, by Scheinin and Makinen $\boldsymbol{e t}$ al. They compared the effects of chronic consumption of sucrose, fructose and Xylitol on dental caries increment, plaque formation and plaque flora. A dramatic reduction in incidence of dental caries was found after 2 years of Xylitol consumption. ${ }^{3}$

\section{Role of Xylitol in Dental Caries:}

Oral microorganisms and specially S. mutans do not have enzymes to utilize Xylitol as a source of energy for acid production or for synthesis of extracellular polysaccharides. Experimental studies in rats have demonstrated an extremely low caries rate in the presence of a Xylitol-containing diet. There are even indications that Xylitol can exert an anti-cariogenic effect. (Edgar WM et al. (1998)

\section{Side effects of xylitol:}

In general, it is assured that side effects of Xylitol rarely occur in people who consume it. This sweetener is recommended as a healthy alternative to sugar for most dieticians. However, it is known that exceeding the 6 to 8 grams of recommended Xylitol for oral care can cause stomach discomfort and above 40 grams a day, it might cause diarrhoea. ${ }^{1}$

\subsection{MALTITOL:}

This organic product is derived from a number of plant materials (bark of Larch trees, pine needles, chicory, and roasted malt) and is manufactured and sold in pure form.

In dental experimental studies, it was seen that some strains of S. Sanguis and S. Mutans were able to ferment maltitol and resulted in weak acid production. The acid production rate was however low. Also S.mutans were unable to synthesize intracellular polysaccharides from maltitol. Thus, the present knowledge gained from various types of microbiological studies, indicate that maltitol may be an alternative sugar substitute. (Birkhed et al.(1985). It has been used to replace table sugar because it has fewer calories, does not promote tooth decay and has a somewhat lesser effect on blood glucose. ${ }^{2}$

\section{Side effects of maltitol:}

Due to its slow absorption, excessive consumption can have laxative effect and sometimes can cause gas and/or bloating. Maltitol is particularly associated with gastric distress. ${ }^{2}$

\section{I.2 STARCH HYDROSYLATES}

\section{I.2A Lycasin:}

Lycasin is a hydrogenated starch hydrolysate, which consist of mixture of sorbitol, maltitol and hydrogenated dextrins of various molecular weights. ${ }^{2}$

From a series of rat experiments, in which the animals had been inoculated with S. mutans or a combination of S. mutans and a viscosus, it was found that lycasin could be classified as being non-cariogenic, no adaptation of practical importance develops in dental plaque to lycasin and combination of xylitol and lycasin also has anticariogenic properties. [Havenaar (1985), Stig Edwardsson et al. (1977)], compared the acid production of Xylitol, lycasin, sorbitol by oral streptococci and lactobacilli with that of sucrose and found 
the acid production of these substitutes to be very less indicating their anticariogenic potential. Also only few strains of bacteria could ferment the sugar substitutes. ${ }^{2}$

\section{I.2B Palatinit: \\ Palatinit}

Palatinit is an equimolar mixture of disaccharide alcohols. The compound has been approved under the name Isomalt by the British Pharmacopoeia Commission. ${ }^{2}$

Some strains of S. mutans, A. Israeli, A. viscosus, and L. caesei are able to ferment Palatinit in vitro, lowering the $\mathrm{pH}$ to levels less than 5.5. Like other polyols, the rate of degeneration is presumably slower than the sucrose. Strains of S. mutans are unable to produce extracellular polysaccharides from Palatinit (Karle $\&$ Gehring(1978). The available microbiological data indicates that palatinit has characteristics similar to other commonly used sugar substitutes, such as sorbitol. ${ }^{2}$

\section{I.3 Coupling Sugars:}

By enzymatic treatment of starch, a mixture of monosaccharides, glucosyl-sucrose, maltosyl-sucrose and oligosaccharides can be produced, called coupling sugars. In Japan it is at present used in sweet and other sweetened foods. Streptococci, Lactobacilli, Actinomyces and Propionibacteria ferment coupling sugar to varying degree. The acid production rate for these strains is 11-54 percent less than that from glucose. The amount of insoluble glucan formed by S. mutans in vitro is approximately 10 percent less compared to sucrose suggesting the lower cariogenic potential of these substitutes as compared to sucrose.

\section{I.3A Sorbose:}

L-Sorbose, an isomer of D-fructose, is a reducing sugar; a ketohexose and belongs to the group of sugars known as monosaccharides.

The low fermentability of Sorbose by bacteria was observed more than 50 years ago. Few oral bacteria are able to ferment Sorbose to acid. S. mutans does not ferment Sorbose. Strains of S. mutans are unable to form intracellular polysaccharides in the presence of Sorbose [Havenaar et al.(1979)]. ${ }^{2}$

\section{I.3B Palatinose:}

Palatinose (isomaltulose) is a sucrose derivative, which is produced by enzymatic conversion of sucrose from a glucosyl- $(1 \rightarrow 2)$ fructoside to a glucosyl- $(1 \rightarrow 6)$-fructoside.

Palatinose and other sucrose isomers have been found not to be utilized by most oral bacteria. Strains of streptococcus mutans did not ferment Palatinose significantly even after 48 hour incubation of the organisms.

\section{I.3C Fructose and Invert Sugar:}

The monosaccharides glucose and fructose and the mixture of them (invert sugar) may be less cariesinducing than sucrose (Scheinin(1979). Fructose and invert sugar are used as sucrose substitutes in the Scandinavian countries.

Bacteriological and biochemical studies of fermentation by oral microorganisms and dental plaque show that acid production from glucose, fructose and invert sugar is of the same order of magnitude as that from sucrose.

\section{II.A CYCLAMATE}

\section{Non-Nutritive /Non-Calorigenic Sugar Substitutes.}

Cyclamate is an artificial sweetener that was discovered in 1937 at the University of Illinois by graduate student Michael Sveda.

Cyclamate was marketed in tablet form for use by diabetics as an alternative tabletop sweetener, as well as in a liquid form.

Cyclamate is $30-50$ times sweeter than sugar (depending on concentration; it is not a linear relationship), making it the least potent of the commercially used artificial sweeteners. Some people find it to have an unpleasant aftertaste, but generally less so than saccharin or acesulfame potassium. ${ }^{16}$

\section{Role of Cyclamate on Plaque and Dental Caries}

The effect of mouth rinses containing cyclamate, Xylitol and sucrose sweeteners on the oral health were evaluated by Paunia K et al. (1984). A simultaneous neglect of oral hygiene was maintained and subjects were made to rinse their mouth six times a day with $15 \mathrm{ml}$ of the respective sweetener. An increase in gingivitis was seen in all the groups. However, less dental plaque was observed in cyclamate and Xylitol groups as compared to sucrose group. 
Side effects of cyclamate:

1. Cyclamate has been associated with bladder cancer which led to its ban in United States in 1970, which could not be substantiated by further studies. However, it is being used as a sugar substitute in many countries.

2. Some experiments on rodents have shown cyclamate to cause testicular atrophy and affect seminal vesicle functions thus causing a negative impact on male reproduction. ${ }^{16}$

\section{II.B SACCHARIN}

Saccharin has been widely used as a sweetener for almost a century. It is an aromatic organic compound, osulfobenzimide $\left(\mathrm{C}_{7} \mathrm{H}_{5} \mathrm{NO}_{3} \mathrm{~S}\right)$. Saccharin was first produced in 1879 by Constantin Fahlberg, a chemist. Although saccharin was commercialized not long after its discovery, it was not until sugar shortages during World War I that its use became widespread. ${ }^{20}$

\section{Role of Saccharin on Dental Caries:}

J. M Tanzer (1988), in an experiment studied the effect of bicarbonate based dental powder containing fluoride and saccharin on inhibition of dental caries associated with S. mutans in rats in comparison to the application of topical fluoride and use of fluoride with de-ionized water. In his results dental powder containing fluoride and saccharin showed maximum reduction in dental caries as compared to topical fluoride alone or when used with de-ionized water.

\section{Side effects of saccharin:}

Throughout the $1960 \mathrm{~s}$, various studies suggested that saccharin might be an animal carcinogen. Concern peaked in 1977, after the publication of a study indicating an increased rate of bladder cancer in rats fed large doses of saccharin. However, U.S. National Institute for Environmental Health Sciences came to the conclusion in 2000, recommending that saccharin be removed from the list of known or suspected human carcinogens. Still, saccharin products are made to carry health warning labels. ${ }^{1}$

\section{Ii.C Aspartame}

Aspartame was discovered in 1965 by James M. Schlatter, a chemist working for G.D. Searle \& Company. It was approved by FDA in 1981. It is widely used in foods and beverages because its taste is very close to that of table sugar. Aspartame is approximately 180 times as sweet as sugar. ${ }^{21}$

\section{Role of Aspartame on Dental Caries}

Das S et al. (1991) conducted a study to test the effect of aspartame alone and along with sucrose, on dental caries in rats. In their findings they discovered animals fed with aspartame alone showed reduced S. mutans count and also dental caries.

\section{Side effects of aspartame:}

Aspartame has been the subject of controversy regarding its safety and the circumstances of its approval by the American FDA. Some studies have also recommended further investigation into possible connections between aspartame and negative effects such as headaches, brain tumors, brain lesions, and lymphoma. ${ }^{21}$

\section{Ii.D Acesulfame Potassium}

Acesulfame potassium is a calorie-free artificial sweetener, also known as Acesulfame $\mathrm{K}$ or Ace $\mathrm{K}$ (K being the symbol for potassium). It was discovered accidentally in 1967 by German chemist Karl Clauss.

Acesulfame K is 180-200 times sweeter than sucrose (table sugar), as sweet as aspartame, about half as sweet as saccharin, and one-quarter as sweet as sucralose.

\section{Side effects of acesulfame potassium:}

Critics of the use of acesulfame potassium say the chemical has not been studied adequately and may be carcinogenic, although these claims have been dismissed by the US FDA and by equivalent authorities in the European Union.

\section{Ii.E Sucralose}




\section{Sucralose \\ Introduction}

Sucralose was discovered in $\mathbf{1 9 7 6}$ by scientists Tate \& Lyle, working with researchers Leslie Hough and Shashikant Phadnis at Queen Elizabeth College. It was first approved for use in Canada (marketed as Splenda) in 1991. Subsequent approvals came in Australia in 1993, in New Zealand in 1996, in the United States in 1998, and in the European Union in 2004. As of 2008, it had been approved in over 80 countries, including Mexico, Brazil, China, India and japan.

\section{Role of Sucralose in Dental Caries}

Sucralose is a new type of non-caloric, high-intensity sweetener recently approved for use by the U.S. FDA. Its availability may expand the number of palatable, low-sugar foods and beverages currently in the market. A series of studies has been conducted to assess whether sucralose has cariogenic potential. The sum of these studies demonstrates that sucralose is non-cariogenic. Steinberg LM (1995) conducted a study to compare the effect on in vivo plaque $\mathrm{pH}$ after rinsing with an aqueous solution of sucralose to the effect of rinsing with an aqueous solution of sucrose. The results indicated that rinsing with aqueous solutions of sucralose was less acidogenic than rinsing with a sucrose solution of equivalent sweetness. ${ }^{8}$ Thus, when used to replace sugar, both sucralose and the tested sucralose-based sweeteners may be useful in the dietary management of caries. (Mandel ID, Grotz VL(2002).

\section{Side Effects of Sucralose:}

Sucralose is said to act as a triggering agent for migraine, also it may decrease the mean weight of the thymus thus affecting the immunity. It is known to cause weight gain and cause some allergic reactions.

\section{Sugar Substitutes Derived From Plant Sources}

Natural products or plant sources are always considered to be better as compared to chemically derived sweeteners as they are free from any chemical mutagen, carcinogen or toxic by-product. ${ }^{1}$

\section{Monellin}

The grape like red berry cluster of serendipity plant (dioscoreophyllum cumminsii, diels) indigenous to Africa are intensely sweet. The active principle is a protein called monellin. The relative sweetness of monellin varies from 800 to 2000 times sweeter than sucrose. Monellin has a slow onset of sweetness and lingering aftertaste. Monellin sweetness is $\mathrm{pH}$ dependent; the protein is tasteless below $\mathrm{pH} 2$ and above $\mathrm{pH} 9 .{ }^{1}$

\section{Side effects:}

It has been accepted in a number of countries and no known side effects have been reported.

\section{Licorice}

This is a well known flavouring agent that is obtained from roots of a small shrub grown in Central Asia and Europe. The roots contain glycyrrhizin, the active sweetener in the form of salts of glycyrrhizin acid. Ammonium glycyrrhizin is commercially available and is about 50 times as sweet as sucrose. It has excellent foaming, flavouring-reinforcement properties and is used in beverages, desserts, dentifrices, and pharmaceutical preparations. $^{1}$

\section{Stevia:}

The species Stevia rebaudiana Bertoni, commonly known as

sweet leaf, sugarleaf, or simply stevia, is widely grown for its sweet leaves. As a sugar substitute, stevia's taste has a slower onset and longer duration than that of sugar, although some of its extracts may have a bitter or licorice -like aftertaste at high concentrations.

With its extracts having up to 300 times the sweetness of sugar, stevia has garnered attention with the rise in demand for low-carbohydrate, low-sugar food alternatives. ${ }^{1}$

\section{TRADE NAMES: Rebiana, truviaSide Effects of Stevia:}

However, health and political controversies have limited stevia's availability in many countries; for example, the United States banned it in the early 1990s unless labeled as a supplement. Stevia is widely used as a sweetener in Japan, and it is now available in the US and Canada as a dietary supplement, although not as a food additive.

\section{Health Controversies Of Sugar Substitute}

As new products are launched in the market controversies are always associated with them. The Primary requisite of products like sugar substitutes, which are meant for human consumption should be free 
from any health risks and should be non toxic to human body. Some of the sweeteners still await their approval from the FDA and research is still going on. ${ }^{1}$ A brief review of some of the hassles associated with various sweeteners is as follows:

\section{Cyclamate controversy:}

In the United States, the Food and Drug Administration banned the sale of cyclamate in 1970 after lab tests indicated that large amounts of cyclamates caused bladder cancer in rats. ${ }^{1}$

\section{Saccharin Controversy:}

There have been worries about the safety of saccharin since its introduction. Fear about the use of saccharin increased when a 1960 study showed that high levels of saccharin may cause bladder cancer in lab rats. In 1977, Canada banned saccharin due to such results from animal research. The FDA in the United States considered banning saccharin in $1977 .{ }^{1}$

\section{Aspartame controversy:}

Aspartame was originally approved for use in July 1974 by the FDA as a nutritive sweetener. During the review period following the initial approval, objections were filed. In December 1975 the FDA retracted its aspartame approval pending a more detailed inspection of the manufacturer's research and public hearings. ${ }^{21}$

It is approved as a free flowing sugar substitute for table use, and for use as cold cereals, drink mixes, instant coffee, instant tea, soft drinks, gelatins, puddings, pie fillings, toppings, dairy products, multivitamin food supplements, and other products where the standards of identity do not preclude such use. The commissioner of the FDA concluded his statement on aspartame before the committee on labor and human resources of the United States senate by stating we do not have any medical or scientific evidence that undermines our confidence in safety of aspartame. However People with phenylketonuria (PKU) should avoid the intake of aspartame because of its phenylalanine content. ${ }^{21}$ Hence all Products sweetened with aspartame must be labelled with the statement phenylketonurics: contains phenylalanine.

\section{Sucralose Controversy:}

The FDA approved sucralose in 1998. The first significant rumblings were in 2000, when Dr. Joseph Mercola wrote an article entitled "The Secret Dangers of Splenda (Sucralose), an Artificial Sweetener". Sucralose is said to act as a trigger for migraine headache, it may decrease the mean weight of the thymus thus affecting the immunity, it's known to cause weight increase and cause some allergic reactions. ${ }^{1}$

\section{Stevia controversy:}

A 1985 study reported that stevia, a breakdown product from stevia side (two of the sweet steviol glycosides in the stevia leaf), is a mutagen in the presence of a liver extract of pre-treated rats but this finding has been criticized on procedural grounds that the data was mishandled in such a way that even distilled water would appear mutagenic. More recent animal tests have shown mixed results in terms of toxicology and adverse effects of stevia extract, with some tests finding stevia to be a weak mutagen while newer studies find no safety issues. ${ }^{1}$

\section{Future prospective of sugar substitutes}

It is now an established fact that sugar definitely contributes to formation of dental cavities. Thus, to prevent dental caries sugar consumption needs to be restricted. However, in view of human taste preference for sweeteners, it is unlikely that many people will voluntarily restrict their sucrose consumption permanently in order to reduce dental caries. Hence, a need arises to replace dietary sugars with substances which provide sweetness but lack the cariogenic effects. For this reason various investigators have searched for alternative sweeteners or sugar substitutes. ${ }^{1}$

A large number of sugar substitutes are available in the market and are fast replacing sugars. These are being added to chewing gums, puddings, ice-creams, desserts, fruit spread, soft-drinks and also being used as a table top sweetener.

Sugar substitutes have marked their role in the dental industry also, as they are being frequently used in tooth-pastes, mouthwashes, mouth-fresheners and chewing gums. ${ }^{1}$ The future prospects are bright for sugar substitutes accounting to the fast pace with which they are replacing natural sugars. However, the use should be within limits in view of their potential side-effects.

\section{New Noncaloric Sweeteners}

Recently many sweeteners have been submitted to the FDA for approval in the United States. Two of the more promising ones are Alitame and Sweetener 2000. ${ }^{1}$ 
Alitame is 2000 times sweeter than sucrose. It is composed of two amino acids, L-aspartic acid and Dalanine. It is metabolized in the body; however, because it's intense sweetness, the caloric contribution to the diet is insignificant. It has a synergistic effect with other sweeteners. Alitame has a clean taste and is stable both at high temperatures and broad $\mathrm{pH}$ ranges. ${ }^{1}$

\section{Conclusion}

There is a considerable amount of information on the effect of non-sugar sweeteners on dental health. This comes from various types of studies like incubation experiments, plaque $\mathrm{pH}$ studies, enamel scab experiments, animal studies, and human clinical trials.

Xylitol has been subjected to many studies of different types and can be classified as noncariogenic. There is evidence that xylitol has a positive anti-cariogenic effect within dental plaque, but it is premature to label it anti-cariogenic other than its action as a non-cariogenic salivary stimulant, thus encouraging remineralization of incipient carious lesions

The evidence for casual relationship between sugars and dental caries has been established. Dental caries still remains a very costly and widespread disease that in many industrialized countries affects mainly disadvantaged individuals and is of serious concern in many developing countries

\section{Recommendations}

- Some dentists do not consider that sugar substitutes are of great importance in a preventive dentistry program. However in the best interest of the patients they should recommend sugar substitutes especially for patients with rampant caries etc.

- Dental research workers should be encouraged to investigate the properties of newer non-sugar sweeteners so that, if they have favourable dental properties, their approval may be quickened and their use encouraged.

- Chewing gum containing protective substance is effective and has the potential to significantly improve oral health status. Policy makers should consider the public health benefits of chewing gum containing dental protective substances especially xylitol.

- The consumers need to be aware of the polyols and similar substances used as bulk sugar substitutes in the food products so, that they can limit their intake sufficiently to avoid any health discomforts.

- The availability of a variety of safe sugar substitutes could be benefit to consumers as it enables food manufacturers to formulate a variety of good testing sweet foods and beverages that are safe for the teeth and lower in caloric content than sugar sweetened foods and beverages.

- Policy-makers should adopt such measures for promoting caries free or caries reducing or caries inert sugars(noncariogenic)

- Public health dentistry has an important role by making lobby effort for promotion of these sugars and also to educate common people the good facts about the alternate sweeteners or sugar substitutes

\section{References}

[1]. Gordon Nikiforuk. Understanding dental caries. Karger publishing company: 204-22.

[2]. Norman O. Harris: Primary Preventive Dentistry $6^{\text {th }}$ edition; 399-418.

[3]. Ernest Newbrun. Sugar substitutes and noncaloric sweetening agent.1989; cariology $3^{\text {rd }}$ Edition; quintessence publishing; pp.136166.

[4]. Brian A. Burt The Future of the Caries Decline. Journal of Public Health Dentistry. 1985; 45 (4), $261-269$.

[5]. Isokangas P. Xylitol chewing gum in caries prevention. A longitudinal study on Finnish school children. Thesis, Proceedings of the Finnish Dental Society.1987; 83: (I) 1-117.

[6]. Paunia K. Effects of sugar alternatives on caries. J Dent Hyg. 1984; 99(8):345-47.

[7]. Tanzer JM. Xylitol Chewing Gum and Dental caries. Int Dent J. 1985; 35:58-65.

[8]. Das S. Aspartame and dental caries in rat. Pediatr Dent.1991; 13(4):217-20.

[9]. Steinberg LM. Remineralising potential, antiplaque, antigingivitis effects of xylitol and sorbitol sweetened chewing gum. Clin. Prev Dent 1992 Sept-Oct; 14(5)31-4

[10]. Kleinberg I. Oral Effects of Sugars and Sweeteners. Int Dent J. 1985; 35: 180-189.

[11]. Makinen KK. Totally rehardened (remineralized) after use of xylitol/sorbitol gum. Acta odontol scand.1998; 56(3):148-56.

[12]. Edgar, WM. Sugar substitutes, chewing gum and dental caries- a review. Br Dent J .1998; 184(1) 29-32.

[13]. Gales MA, Nguyen T-M. Sorbitol compared with xylitol in prevention of dental caries. Ann Pharmacother. 2000; 34:98-10.

[14]. Hildebrandt GH, Sparks BS. Maintaining mutans streptococci suppression with xylitol chewing gum. J Am Dent Assoc. 2000; 131(7):909-16.

[15]. Makinen KK. Efficacy of xylitol in reducing plaque. J Dent Educ.2001; 65(10):1106-9.

[16]. Maguire and A. J. Rugg-Gunn Xylitol and caries prevention- is it a magic bullet? Br Dent J .2003; 194(8): 429-436.

[17]. Christina R. W, Joseph B, and Linda A. McCauley. The Potential Toxicity of Artificial Sweeteners. AAOHN Journal. June 2008, vol. 56,(6) :251-259.

[18]. Roshan N.M,Sakeenabi B.Sugar Substitutes-Reality check: A Review. J Indian Assoc Public Health Dent.2010; 16; 111-120.

[19]. Frostell G. Effect of various sugars and sugar-substitutes on dental caries in hamsters and rats. J Nutr. 1967; 93:65-76.

[20]. Birkhed D. Sugar substitutes - one consequence of the Vipeholm Study? Eur J of Oral Sci .1989; 97 (2): $126-129$.

[21]. Burt BA. Relative consumption of sucrose and other sugars: has it been a factor in reduced caries experience? Caries res. 1993; 27:56-63. 